\title{
A patient with epilepsy suffering from repeatedly hyperbilirubinemia caused by Gilbert Syndrome: a case report and literatures review
}

\section{Yao-yao Zhang}

Xijing Hospital Department of Neurology

\section{Yong-li Jiang}

Xijing Hospital Department of Neurology

\section{Chang-geng Song}

Xijing Hospital Department of Neurology

\section{Zhi-han Zhao}

Xijing Hospital Department of Neurology

\section{Fang Yuan}

Xijing Hospital Department of Neurology

\section{Fang Yang}

Xijing Hospital Department of Neurology

Wen Jiang ( $\nabla$ jiangwen@fmmu.edu.cn )

Xijing Hospital Department of Neurology

\section{Case Report}

Keywords: Epilepsy, Gilbert syndrome, Valproic acid, Lamotrigine, Levetiracetam, hyperbilirubinemia

Posted Date: January 23rd, 2019

DOI: https://doi.org/10.21203/rs.2.244/v1

License: (c) (i) This work is licensed under a Creative Commons Attribution 4.0 International License. Read Full License 


\section{Abstract}

Background: Certain antiepileptic drugs (AEDs) such as valproic acid (VPA) and lamotrigine (LTG) would bring some abnormalities on liver function, ranging from mild malfunction of liver tests to serious hepatotoxicity. The former manifests temporary and reversible elevation of bilirubin and liver enzyme, which is usually dose-dependent and the abnormality can return to normal shortly after the drug was withdrawn. Levetiracetam (LEV), as a newly broad-spectrum AED with relatively fewer side-effects and fewer drug interactions, is increasingly used as adjunctive therapy to treat generalized epilepsy. Gilbert syndrome $\triangle \mathrm{GS} \otimes i$ is characterized by mild, chronic, intermittent unconjugated hyperbilirubinemia without other hepatic diseases. Fasting, stress, along with some liver toxic drugs would make it clinically apparent. However, when GS co-exists with epilepsy, how to differentiate which one is responsible for the abnormality of bilirubin poses a challenge to us. We herein describes a patient with epilepsy complicated by mildly and intermittently elevated bilirubin, and after various examinations especially for whole-exome sequencing $₫$ WES $\llbracket$ and liver biopsy, we made a definite diagnosis of GS.

Case presentation: A 25-year-old male with epilepsy suffering from intermittently and mildly elevated bilirubin, and relevant imaging information led us consider the diagnosis of idiopathic generalized epilepsy (IGE). After administration of AEDs, the indirect bilirubin remained still slightly elevated even while taking LEV. Then he was referred to the department of gastroenterology in our hospital, the results of diagnostic tests, clinical manifestation, imaging studies, WES and liver biopsy all made contributions to our diagnosis of GS rather than the hepatic injury induced by AEDs.

Conclusions: This report presents us with a case of an epilepsy patient complicated by repeatedly elevated bilirubin and finally was diagnosed with GS. By referring to literatures and genetic testing, though, no shared genetic and pathophysiological basis between epilepsy and GS was found, and enzymes responsible for the metabolism of AEDs took no part in the pathogenesis of GS. Our experiences will help clinicians to better differentiate the etiology of repeatedly elevated bilirubin of epilepsy patients during the process of treatment.

Key words: Epilepsy, Gilbert syndrome, Valproic acid, Lamotrigine, Levetiracetam, hyperbilirubinemia.

\section{Background}

Epilepsy is one of the most common chronic neurological disorders, caused by heterogeneous etiologies involving genetic, structural, infectious, metabolic, immune and other unknown factors, with an enduring predisposition to engender unprovoked epileptic seizures [1]. Gilbert syndrome (GS) , a benign and hereditary hyperbilirubinemia, is characterized by intermittent indirect bilirubin elevation which is attributed to the reduced activity of UDP-glucuronosyl transferase 1A isoform 1 (UGT1A1) [2]. With male more vulnerable than female, it has a prevalence of $3 \%-10 \%$ of the general population $[3,4]$.

It has been known that certain antiepileptic drugs (AEDs) such as valproic acid (VPA) and lamotrigine (LTG) would give rise to the hepatic injury, ranging from mild and reversible abnormality of liver function 
tests to serious hepatotoxicity [5, 6]. However, Levetiracetam (LEV), which is metabolized by enzymatic hydrolysis of the acetamide group in blood rather than liver cytochrome P450 enzymes, and excreted by the urinary system $[7,8]$, has a minor effect on liver function. The combination of minor influence on liver and good seizure control in idiopathic generalized epilepsy (IGE) make LEV a better choice for epilepsy accompanied by GS, though its efficacy in seizure control of IGE ranks only third to VPA and LTG [9].

\section{Case Presentation}

Afterwards, he was referred to the outpatient department of Xijing hospital. Taking hepatic injury induced by VPA into account, we switched the medication to lamotrigine (LTG) and the dosage was based over 6 weeks, from $25 \mathrm{mg}$ qd to $50 \mathrm{mg}$ bid. However, the patient's bilirubin remained still elevated mildly and intermittently, thus we chose the alternative drug LEV as the most appropriate medicine, and its dosage was based over 2 weeks, from $250 \mathrm{mg}$ bid to $500 \mathrm{mg}$ bid while LTG was withdrawn gradually. In the meantime, he was recommended to consult in the department of gastroenterology to identify the definitive cause of mild hyperbilirubinemia.

In July 2011, an adjustment of the dosage of LEV was made after another epileptic seizure occured. In the meanwhile, he was admitted to the department of gastroenterology in Xijing hospital for intermittently elevated bilirubin. The liver function tests showed that TBIL $41.1 \mu \mathrm{mol} / \mathrm{L} \otimes$ normal $\otimes 3.4 \sim 20.5 \mu \mathrm{mol} / \mathrm{L}), \mathrm{DBIL}$ $12.7 \mu \mathrm{mol} / \mathrm{L}$ (normal: 1.7 6.8 $\mu \mathrm{mol} / \mathrm{L}$ ), IBIL $28.4 \mu \mathrm{mol} / \mathrm{L} \bigotimes$ normal $\varangle 6.8 \sim 12.0 \mu \mathrm{mol} / \mathrm{L} \rrbracket$, while there was no jaundice in his skin and mucosa. The tests for Hepatitis $A, B$, and $C$ in serum were negative and the abdominal ultrasound was normal. Diagnostic tests including caloric restriction, phenobarbital and oral phenytoin sodium stimulation were performed and the results were all positive (Table 1), which demenstrated that he was clinically suspected of GS. For the sake of further substantiating our hypothesis, we conducted a whole-exome sequencing (WES). The results showed that causative genetic mutation of epilepsy was negative while a homozygous mutation ( $p$. A(TA)6TAA ins TA) in the UGT1A1 promoter of GS pathogenic gene locus was found (Figure 1). Having completed the above-mentioned tests, the patient was diagnosed as IGE accompanied by GS. In order to exclude the liver damage induced by VPA and/or LTG and confirm the diagnosis of GS, we conducted a liver biopsy showing that there was no apparent abnormality. Ultimately, the diagnosis of GS was established.

He was subsequently discharged from the hospital and was suggested to avoid hunger, fatigue and the usage of VPA, LTG or some other drugs which were metabolized by hepatic glucoronidation. After that, his seizures were controlled with $500 \mathrm{mg}$ of LEV per day but complicated by the occurrence of intermittent hyperbilirubinemia within the next 7 years. In 2017, another 24h-V-EEG was conducted and the result showed no abnomality. At present, the patient has already stopped taking any AEDs and the liver test results for the next 2, 5 and 11 months after the withdrawal of AEDs were shown as the following, respectively (Table 1): TBIL $50.9 \mu \mathrm{mol} / \mathrm{L}$ (normal: 0 25 $\mu \mathrm{mol} / \mathrm{L}$ ), DBIL $17.5 \mu \mathrm{mol} / \mathrm{L}$ (normal: $0 \sim 7 \mu \mathrm{mol} / \mathrm{L}$ ), IBIL $33.4 \mu \mathrm{mol} / \mathrm{L}$ (normal: 0 18 $\mu \mathrm{mol} / \mathrm{L}$ ); TBIL $24.4 \mu \mathrm{mol} / \mathrm{L}$, DBIL $6.9 \mu \mathrm{mol} / \mathrm{L}$, IBIL $17.5 \mu \mathrm{mol} / \mathrm{L} ;$ TBIL $39.1 \mu \mathrm{mol} / \mathrm{L}, \mathrm{DBIL} 11.2 \mu \mathrm{mol} / \mathrm{L}$, IBIL $27.9 \mu \mathrm{mol} / \mathrm{L}$. 


\section{Discussion And Conclusions}

The case demonstrated that a young male with epilepsy suffering from intermittently and mildly elevated bilirubin caused by GS. GS is characterized by mild, chronic, intermittent unconjugated hyperbilirubinemia without other hepatic diseases or overt hemolysis. Fasting, physical exercise, stress, along with some liver toxic drugs would make it clinically apparent, but treatment is typically not necessary [10]. Its pathogenesis involves the reduced activity of UGT1A1 [2], a phase冈drug metabolic enzyme, which is primarily capable of converting a series of insoluble drugs such as irinotecan and atazanavir $[11,12]$ to water-soluble substances which are easy for the renal or biliary elimination and excretion. In addition, it is also the isozyme that plays a significant role in bilirubin conjugation. The activity of UGT1A1 can be reduced by $70 \%$ of the normal level among these patients [13], which results in intermittently and mildly elevated unconjugated bilirubin, mostly ranging from 20 to $50 \mu \mathrm{mol} / \mathrm{L}$ but rarely exceeds $70 \mu \mathrm{mol} / \mathrm{L}[14$, 15].

Currently, the diagnosis of GS is usually one of exclusion, based on mild elevation of serum indirect bilirubin in the absence of other reasons of unconjugated hyperbilirubinemia $[15,16]$. Physical examination, biochemistry tests, imaging studies and liver histology (usually not necessary) are also found to be normal in GS. Notably, genetic detection is playing an increasingly decisive role in differentiating GS from other hereditary hyperbilirubinemia like Crigler-Najjar syndrome [2]. According to the patient's clinical manifestation, biochemistry of liver function, results of diagnostic tests, abdominal ultrasound, liver biopsy and WES, other causative factors of elevated bilirubin were excluded.

GS is susceptible to cause accumulative toxicity of certain therapeutic drugs metabolized by the enzyme UGT1A1】such as irinotecan and atazanav [17-19]. VPA and LTG are the common drugs for the treatment of generalized epileptic seizures [9]. The metabolism of VPA has an extensive range, $30 \%-40 \%$ of the dose is via conjugation with UDP-glucuronyl transferases (UGT) such as UGT1A3 and UGT2B7 [20], and the pathway of mitochondrial beta oxidation accounts for $40 \%$. Other oxidative pathways such as Omega and omega-1 also are implicated in it [21]. On the other hand, the enzyme UGT1A4 may be involved in the metabolism of LTG [22]. Therefore, GS would not lead to accumulative toxicity of VPA and LTG.

Furthermore, both the drugs would not inhibit the activity of UGT1A1 [23], namely, GS would not be induced by them.

Clarkson et al $[5,6]$ once reported that VPA/LTG can cause liver damage to various degrees, and hepatic injuries associated with these drugs can be classified to two subtypes: common type and fulminant liver failure. The former manifests temporary and reversible elevation of bilirubin and liver enzyme values, which is usually dose-dependent and the abnormality can return to normal shortly after the drug is withdrawn [24]; the latter is life-threatening and infrequent, occurring generally within 2-8 weeks (LTG) or 3 months (VPA) after initiating the use of LTG/VPA $[25,26]$. In general, VPA side-effects would not manifest the symptoms of hypersensitivity like LTG but rather more characterized by signs which are quite similar to GS: fatigue, nausea, and even continuous vomiting [27]. That may cause confusion when we 
diagnosed the patient as GS. Despite the fact that VPA and LTG can engender abnormalities of liver tests, there was no concensus about the concrete figure of bilirubin elevation by consulting literatures.

In this case, we did not withdraw LTG immediately, considering the fact that the harm caused by epileptic seizures was much more severe than the signs of mild abnormal liver tests possibly induced by LTG. Furthermore, the patient was not diagnosed with hypersensitivity to LTG in that there was no fever, rash, eosinophilia, and evidence of multiple organ dysfunction during the treatment period [28].

However, after administration of AEDs, the bilirubin still showed slightly abnormal. We substituted LTG with LEV given that LEV, as a newly broad-spectrum AED with relatively fewer side-effects and fewer drug interactions, is increasingly used as adjunctive therapy to treat generalized epilepsy [29]. Moreover, it doesn't inhibit the activity of UGT1A1, and about $34 \%$ of its dose is metabolized via enzymatic hydrolysis of the acetamide group in blood rather than liver cytochrome P450 enzymes, and the remainder is excreted in urine $[7,8]$. Then the patient was clinically suspected of GS based on the diagnostic tests and the clinical manifestation after discontinuation of LTG while taking LEV. Furthermore, his WES and liver biopsy substantiated our assumption. Even after discontinuing LEV, the indirect bilirubin were still high intermittently. Up to now, there were, to our knowledge, almost no reports of bilirubin abnormalities caused by LEV monotherapy. For a patient with epilepsy suffering from repeatedly elevated bilirubin, timely diagnosis, treatment and periodical liver tests are necessary for choosing appropriate AEDs, especially when it co-exists with GS.

\section{Abbreviations}

GS: Gilbert syndrome; UGT1A1: UDP-glucuronosyl transferase 1A isoform 1; AEDs: Antiepileptic drugs; VPA: Valproic acid; LTG: Lamotrigine; LEV: Levetiracetam; IGE: Idiopathic generalized epilepsy; EEG: Electroencephalogram; MRI: Magnetic resonance imaging; WES: Whole-exome sequencing; TBil: Total bilirubin; DBil: Direct bilirubin; IDBil: Indirect bilirubin; UGT: UDP-glucuronyl transferases

\section{Declarations}

This case report is in accordance with BioMed Central editorial policies (http://www.biomedcentral.com/submissions/editorial-policies\#standards+of+reporting), and I ensure that my manuscript reporting adheres to CARE guidelines (http://www.care-statement.org/downloads) for reporting of case reports. A completed CARE checklist as an additional one in my submission files.

\section{Ethics approval and consent to participate}

Not applicable. 


\section{Consent to publish}

Written informed consent was obtained from the patient for publication of this Case report and any accompanying images. A copy of the written consent is available for review by the Editor of this journal.

\section{Availability of data and materials}

All data and material supporting our findings are contained within the manuscript.

\section{Competing interests}

The authors declare that they have no conflicts of interest.

\section{Funding}

Not applicable

\section{Authors' contributions}

YZ: acquired and analyzed the raw clinical data, wrote the manuscript, the critical revision, study supervision. YJ: acquisition/analysis/interpretation of data, the critical revision, study supervision. CS: acquisition/analysis/interpretation of data, critical revision, study supervision. ZZ, FY, FY: acquisition/analysis/interpretation of data, critical revision, study supervision. WJ: study concept and design, critical revision, study supervision. All authors read and approved the final manuscript.

\section{Acknowledgements}

We thank the patient for their participation in this research. We also thank the MyGenostics Company for supporting this study.

\section{Authors' information}

Department of Neurology, Xijing Hospital, Fourth Military Medical University, Xi'an, 710032, Shaanxi Province, China 


\section{References}

1. Scheffer IE, Berkovic S, Capovilla G, Connolly MB, French J, Guilhoto L, Hirsch E, Jain S, Mathern GW, Moshe SL et al: ILAE classification of the epilepsies: Position paper of the ILAE Commission for Classification and Terminology. EPILEPSIA 2017, 58(4):512-521.

2. Bosma PJ, Chowdhury JR, Bakker C, Gantla S, de Boer A, Oostra BA, Lindhout D, Tytgat GN, Jansen $\mathrm{PL}$, Oude ER et al: The genetic basis of the reduced expression of bilirubin UDPglucuronosyltransferase 1 in Gilbert's syndrome. N Eng/ J Med 1995, 333(18):1171-1175.

3. Radu P, Atsmon J: Gilbert's syndrome-clinical and pharmacological implications. ISR MED ASSOC J 2001, 3(8):593-598.

4. Radoi VE, Ursu RI, Poenaru E, Arsene C, Bohiltea CL, Bohiltea R: Frequency of the UGT1A1*28 Polymorphism in a Romanian Cohort of Gilbert Syndrome Individuals. J Gastrointestin Liver Dis 2017, 26(1):25-28.

5. Clarkson A, Choonara I: Surveillance for fatal suspected adverse drug reactions in the UK. ARCH DIS CHILD 2002, 87(6):462-466.

6. Su-Yin AN, Tai WW, Olson KR: Lamotrigine-associated reversible severe hepatitis: a case report. $J$ Med Toxicol 2008, 4(4):258-260.

7. Strolin BM, Whomsley R, Nicolas JM, Young C, Baltes E: Pharmacokinetics and metabolism of 14Clevetiracetam, a new antiepileptic agent, in healthy volunteers. EUR J CLIN PHARMACOL 2003, 59(89):621-630.

8. Patsalos PN: Clinical pharmacokinetics of levetiracetam. CLIN PHARMACOKINET 2004, 43(11):707724.

9. Schmidt D, Schachter SC: Drug treatment of epilepsy in adults|. *348*348; 2014.

10. Powell LW, Hemingway E, Billing BH, Sherlock S: Idiopathic unconjugated hyperbilirubinemia (Gilbert's syndrome). A study of 42 families. N Engl J Med 1967, 277(21):1108-1112.

11. Ehmer U, Kalthoff S, Fakundiny B, Pabst B, Freiberg N, Naumann R, Manns MP, Strassburg CP: Gilbert syndrome redefined: a complex genetic haplotype influences the regulation of glucuronidation. HEPATOLOGY 2012, 55(6):1912-1921.

12. Hu Z, Yu Q, Zhao Y: Dosedependent association between UGT1A1 $* 28$ polymorphism and irinotecan-induced diarrhoea: A meta-analysis. EUR J CANCER 2010, 46(10):1856-1865.

13. Cuperus FJ, Hafkamp AM, Hulzebos CV, Verkade HJ: Pharmacological therapies for unconjugated hyperbilirubinemia. Curr Pharm Des 2009, 15(25):2927-2938.

14. Hirschfield GM, Alexander GJ: Gilbert's syndrome: an overview for clinical biochemists. ANN CLIN BIOCHEM 2006, 43(Pt 5):340-343.

15. Erlinger S, Arias IM, Dhumeaux D: Inherited disorders of bilirubin transport and conjugation: new insights into molecular mechanisms and consequences. GASTROENTEROLOGY 2014, 146(7):1625- 
1638.

16. Maruo Y, Nakahara S, Yanagi T, Nomura A, Mimura Y, Matsui K, Sato H, Takeuchi Y: Genotype of UGT1A1 and phenotype correlation between Crigler-Najjar syndrome type II and Gilbert syndrome. J Gastroenterol Hepatol 2016, 31(2):403-408.

17. Horsfall LJ, Nazareth I, Pereira SP, Petersen I: Gilbert's syndrome and the risk of death: a populationbased cohort study. J Gastroenterol Hepatol 2013, 28(10):1643-1647.

18. Lankisch TO, Moebius U, Wehmeier M, Behrens G, Manns MP, Schmidt RE, Strassburg CP: Gilbert's disease and atazanavir: from phenotype to UDP-glucuronosyltransferase haplotype. HEPATOLOGY 2006, 44(5):1324-1332.

19. Lankisch TO, Schulz C, Zwingers T, Erichsen TJ, Manns MP, Heinemann V, Strassburg CP: Gilbert's Syndrome and irinotecan toxicity: combination with UDP-glucuronosyltransferase $1 \mathrm{~A} 7$ variants increases risk. Cancer Epidemiol Biomarkers Prev 2008, 17(3):695-701.

20. Tomson T, Landmark CJ, Battino D: Antiepileptic drug treatment in pregnancy: changes in drug disposition and their clinical implications. EPILEPSIA 2013, 54(3):405-414.

21. Vajda FJ, Eadie MJ: The clinical pharmacology of traditional antiepileptic drugs. EPILEPTIC DISORD 2014, 16(4):395-408.

22. Rowland A: In vitro characterization of lamotrigine N2-glucuronidation and the lamotrigine - valproic acid interaction. DRUG METAB DISPOS 2006.

23. Radlovic N: Hereditary hyperbilirubinemias. Srp Arh Celok Lek 2014, 142(3-4):257-260.

24. Powell-Jackson PR, Tredger JM, Williams R: Hepatotoxicity to sodium valproate: a review. GUT 1984, 25(6):673-681.

25. Im SG, Yoo SH, Park YM, Lee SJ, Jang SK, Jeon DO, Cho HJ, Oh MJ: Liver dysfunction induced by systemic hypersensitivity reaction to lamotrigine: case report. Clin Mol Hepatol 2015, 21(2):180-182.

26. Krahenbuhl S, Brandner S, Kleinle S, Liechti S, Straumann D: Mitochondrial diseases represent a risk factor for valproate-induced fulminant liver failure. Liver 2000, 20(4):346-348.

27. Koenig SA, Buesing D, Longin E, Oehring R, Haussermann P, Kluger G, Lindmayer F, Hanusch R, Degen I, Kuhn $\mathrm{H}$ et al: Valproic acid-induced hepatopathy: nine new fatalities in Germany from 1994 to 2003. EPILEPSIA 2006, 47(12):2027-2031.

28. Bohan KH, Mansuri TF, Wilson NM: Anticonvulsant hypersensitivity syndrome: implications for pharmaceutical care. PHARMACOTHERAPY 2007, 27(10):1425-1439.

29. Berkovic SF, Knowlton RC, Leroy RF, Schiemann J, Falter U: Placebo-controlled study of levetiracetam in idiopathic generalized epilepsy. NEUROLOGY 2007, 69(18):1751-1760.

\section{Figures}




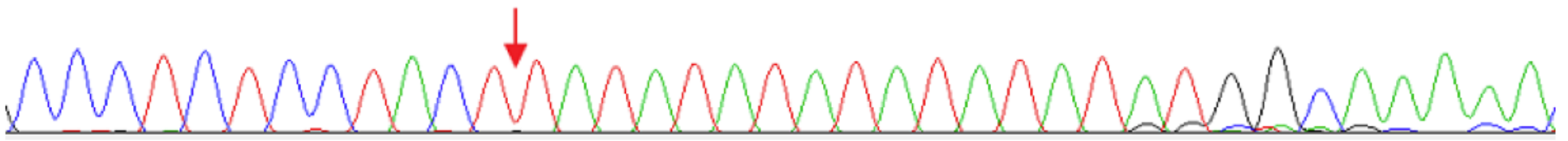

\section{Figure 1}

Homozygous mutation (p. A(TA)6TAA ins TA) in the UGT1A1 promoter of GS pathogenic gene locus: chr2 234668880-234668881*

\section{Supplementary Files}

This is a list of supplementary files associated with this preprint. Click to download.

- CAREChecklist.docx 